\title{
A NEAR-INFRARED, WIDE-FIELD, PROPER-MOTION SEARCH FOR BROWN DWARFS
}

\author{
JoAnnah L. Hinz and Donald W. McCarthy, JR. \\ Steward Observatory, University of Arizona, 933 North Cherry Avenue, Tucson, AZ 85721; jhinz@as.arizona.edu, mccarthy@as.arizona.edu \\ Doug A. Simons \\ Northern Operations Center, Gemini Observatory, 670 North A‘ohoku Place, Hilo, HI 96720; dsimons@gemini.edu \\ TODD J. HENRY \\ Department of Physics and Astronomy, Georgia State University, Atlanta, GA 30303; thenry@chara.gsu.edu \\ J. DAVY KIRKPATRICK \\ Infrared Processing and Analysis Center, MS 100-22, California Institute of Technology, Pasadena, CA 91125; davy@ipac.caltech.edu \\ AND \\ PATRICK C. MCGUIRE \\ Universität of Bielefeld, D-33615 Bielefeld, Germany; mcguire@physik.uni-bielefeld.de \\ Received 2001 October 31; accepted 2002 January 9
}

\begin{abstract}
A common proper-motion survey of M dwarf stars within 8 pc of the Sun reveals no new stellar or brown dwarf companions at wide separations $(\sim 100-1400$ AU). This survey tests whether the brown dwarf "desert" extends to large separations around $\mathrm{M}$ dwarf stars and further explores the census of the solar neighborhood. The sample includes 66 stars north of $-30^{\circ}$ and within $8 \mathrm{pc}$ of the Sun. Existing first-epoch images are compared with new $J$-band images of the same fields an average of $7 \mathrm{yr}$ later to reveal propermotion companions within a $\sim 4^{\prime}$ radius of the primary star. No new companions are detected to a $J$-band limiting magnitude of $\sim 16.5$, corresponding to a companion mass of $\sim 40$ Jupiter masses for an assumed age of $5 \mathrm{Gyr}$ at the mean distance of the objects in the survey, $5.8 \mathrm{pc}$.
\end{abstract}

Key words: stars: imaging — stars: low-mass, brown dwarfs — stars: statistics

\section{INTRODUCTION}

Although the substellar initial mass function (IMF) has been studied in a range of environments, such as starforming clusters (Luhman et al. 1998; Luhman 2000; Najita, Tiede, \& Carr 2000), young open clusters (Bouvier et al. 1998; Barrado y Navascues et al. 2001), and the field (Reid et al. 1999), the IMF of low-mass companions is not well understood, especially at "wide" (greater than 100 AU) separations. Radial velocity searches around solar-type, main-sequence stars (see, e.g., Mayor \& Queloz 1995; Marcy \& Butler 1996) have produced few confirmed brown dwarfs at separations less than 3 AU. Fewer than $0.5 \%$ of their sample have brown dwarf companions at those separations. A coronagraphic search for companions in the range 40-100 AU (Oppenheimer et al. 2001) produced only one brown dwarf, GJ 229B (Nakajima et al. 1995), well below the $17 \%-30 \%$ multiplicity observed for all stars (Reid \& Gizis 1997). Other types of surveys, such as high spatial resolution, space-based observations (Lowrance et al. 1999, 2000) and ground-based adaptive optics (Els et al. 2001), have also resulted in discoveries of low-mass stellar and substellar companions. However, the frequency of stellar and substellar companions at close separations remains distinctly different, resulting in the idea that there is a "brown dwarf desert."

To date, there has been only one systematic search for brown dwarf companions at wide separations and with a volume-limited sample (Simons, Henry, \& Kirkpatrick 1996, hereafter SHK96). This was mainly a color-based search around $\mathrm{M}$ dwarfs within $8 \mathrm{pc}$ of the Sun and did not turn up any new brown dwarfs, although, given the surpris- ingly blue colors of GJ 229B, cool brown dwarfs with intermediate $J-K$ colors may have been overlooked in the survey.

Proper-motion searches for companions have been used for many years to identify low-mass objects (see, e.g., van Biesbroeck 1961) and offer a less biased way of finding lowmass companions than color-based surveys. Therefore, we have conducted the planned second-epoch survey of the SHK96 sample, in order to identify low-mass companions to $\mathrm{M}$ dwarfs at wide separations out to over $1000 \mathrm{AU}$. The choice of M dwarf primaries is significant: Reid \& Gizis (1997) and Reid et al. (1999) show that the distribution of mass ratios for a sample of $80 \% \mathrm{M}$ dwarfs has a peak at $q=0.95$, where $q$ is the ratio of the secondary mass to the primary mass. They conclude that their sample shows a distinct bias toward approximately equal-mass systems and that the mass function for stellar companions is different from the IMF of field stars. If these conclusions extend to brown dwarf masses, $\mathrm{M}$ primaries may harbor more substellar companions than other stellar types. On the other hand, Reipurth \& Clarke (2001) suggest that brown dwarfs have been ejected by dynamical interactions during the star formation process and cannot accrete enough mass to become stars. In this case, $\mathrm{M}$ dwarf primaries may not be accompanied by such companions except in a multiple $\mathrm{M}$ dwarf system with a correspondingly large gravitational potential.

Thus, our proper-motion search around one spectral class of primaries fills a unique niche in the search for low-mass stellar and brown dwarf companions. We describe the data acquisition and reduction in $\S 2$ and discuss the results of the survey in $\S 3$. 


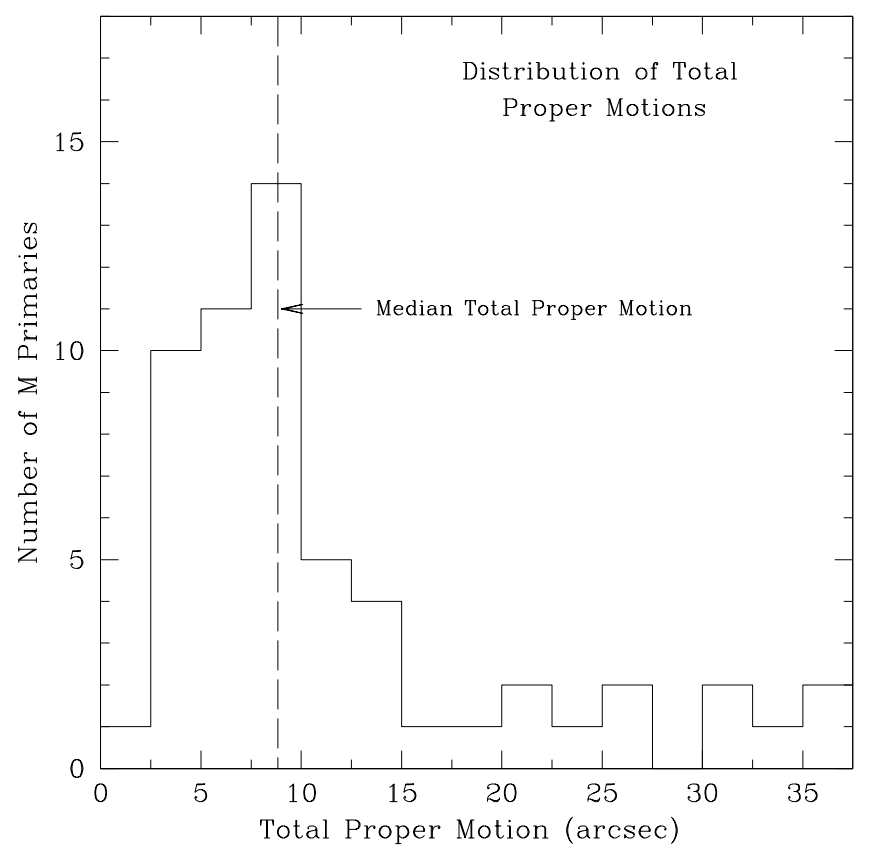

FIG. 1.- Histogram illustrating the distribution of total proper motions between epochs (in arcseconds) of the M dwarfs observed in this survey. The median total proper-motion value is 8 ". 8 , as indicated above.

\section{OBSERVATIONS AND DATA REDUCTION}

\subsection{Sample Selection}

Our sample is identical to Henry's (1991) list of M dwarfs within $8 \mathrm{pc}$ of the Sun. The M dwarfs were chosen initially from the Second Catalog of Nearby Stars (Gliese 1969) and its updates (Gliese \& Jahreiss 1979), along with other additions from more recent literature (e.g., LHS 292; see SHK96 for more details). The sample consists of $75 \mathrm{M}$ dwarf primaries with $M_{V} \geq 8.0 \mathrm{mag}$, trigonometric parallaxes $\geq 0$ ". 125 , and declinations north of $-30^{\circ}$. SHK96 observed 66 of these systems, discarding three because of confusion toward the galactic plane (GJ 701, 729, and 752), leaving a total of 63 systems.

Of the original 75 systems, we observed 74 targets. Table 1 contains a list of the targets, their proper motions, the dates of observation and those of SHK96, and other relevant parameters. The median distance of the $\mathrm{M}$ dwarf targets is $5.8 \mathrm{pc}$, corresponding to a median search radius of $1480 \mathrm{AU}$ in the present survey. Over the complete distance range, the search radius varies from 800 to $2100 \mathrm{AU}$. Figure 1 shows the distribution of total proper motions of our objects between the first- and second-epoch observations. Due to discoveries of new objects and to the measurement of more accurate parallaxes, the SHK96 list is no longer a complete volume-limited sample. Table 1 has five objects from the original sample whose updated parallaxes move them beyond the 8 pc limit (GJ 185, 623, 686, 1230, and 884) and separately lists four objects whose redetermined parallaxes or recent discoveries (e.g., G 180-060; Ducourant et al. 1998) place them within the survey criteria.

\subsection{Imaging}

First-epoch images (SHK96) were obtained at the University of Hawaii's 24 inch $(0.6 \mathrm{~m})$ telescope between
1991 August and 1992 August with a facility $256 \times 256$ NICMOS camera in both the $J$ and $K^{\prime}$ bands with a scale of 2 ." $0 \mathrm{pixel}^{-1}$. Exposure times were typically $1 \mathrm{hr}$, and images were processed using conventional techniques. A custom program searched for point sources above a $3 \sigma$ detection level and performed photometry on all sources using a $10^{\prime \prime}$ aperture.

Between 1998 April and 2000 December, $J$-band images of the same fields were taken with the PISCES camera (McCarthy et al. 2001) at the Bok $2.3 \mathrm{~m}$ Telescope on Kitt Peak. PISCES has an 8.5 diameter field of view and a 0.5 pixel $^{-1}$ plate scale at this telescope. Nine 30 s exposures were obtained, centered on a program $\mathrm{M}$ dwarf, with a $10^{\prime \prime}$ dither between each exposure. All images were corrected for quadrant cross-talk effects known to be present in HAWAII arrays (McCarthy et al. 2001). The images were then darksubtracted, flat-fielded, masked for hot pixels, corrected for geometric distortion, and combined with standard IRAF tasks. The flat field was produced through a median combination of the dithered science frames. The flat field is predictably poor in the region of the bright $\mathrm{M}$ star, which was allowed to saturate the detector. However, the flat fielding does not affect the astrometry of the field, changing the position of the M dwarf by less than half a pixel in multiple test cases. In addition, because accurate photometry of the objects has already been carried out by SHK96, the resulting flat field is adequate for this survey.

Figure 2 shows a sample fully reduced field (GJ 752), along with the identical field observed by SHK96. The field-of-view sizes are almost perfectly matched, except that PISCES has a circular inscribed field. The SHK96 images show an internal reflection due to the optics in the camera to the lower right of the $\mathrm{M}$ dwarf primary that is not in the second-epoch set. The SHK96 survey has a limiting magnitude of $m_{J} \sim 16.5$ and is sensitive to companions down to $40 M_{\text {Jupiter }}$ assuming an age of 5 Gyr at the mean distance of the survey, $5.8 \mathrm{pc}$, based on the models of Burrows et al. (1997). We use $5 \mathrm{Gyr}$, following the findings of Henry (1991) on this sample; however, because age dating $M$ dwarfs is difficult, 5 Gyr may not be an accurate average age. If the $M$ dwarfs are instead $1 \mathrm{Gyr}$ old, the survey is sensitive to $16 M_{\text {Jupiter }}$. The sensitivity of the PISCES images matches or exceeds that of the SHK96 images in all cases with $m_{J} \sim 17.0$.

Each second-epoch image is compared with its matching first-epoch image using an IRAF script originally designed to identify supernovae in nearby galaxies (Van Dyk et al. 2000). Using input coordinates of identical objects in the two frames, the script matches the pixel scales of the two cameras, accounts for any differences in geometric distortion, matches the point-spread functions for the two images, and subtracts them. Typically, 10-15 background stars from each $\mathrm{M}$ dwarf field were used as input references for the program. The total number of background stars ranges from 50 to over 1000 sources for the crowded fields near the galactic plane. The mean total proper motion of the $\mathrm{M}$ dwarfs between epochs is $8.8(\sim 18$ pixels on the PISCES camera). Moving objects are revealed by adjacent positive and negative images. Companion objects would have proper-motion vectors identical to the $\mathrm{M}$ dwarf primaries that have been accurately measured by Hipparcos (Perryman et al. 1997). Objects with such large proper motions can easily be detected by visually examining the subtracted images. 
TABLE 1

Parameter List for Target Program Stars

\begin{tabular}{|c|c|c|c|c|c|c|c|}
\hline $\begin{array}{c}\text { Primary Name } \\
\text { (1) }\end{array}$ & $\begin{array}{l}\text { Components } \\
\text { (2) }\end{array}$ & $\begin{array}{l}\text { Trig. Parallax } \\
\text { (3) }\end{array}$ & $\begin{array}{c}\text { Proper Motion } \\
\text { (4) }\end{array}$ & $\begin{array}{l}\text { First Epoch } \\
\text { (5) }\end{array}$ & $\begin{array}{l}\text { Second Epoch } \\
\text { (6) }\end{array}$ & $\begin{array}{l}M_{V} \\
\text { (7) }\end{array}$ & $\begin{array}{l}\text { Sp. Type } \\
\text { (8) }\end{array}$ \\
\hline GJ 1002. & & $0.2128 \pm 0.0033$ & 2.041 & & 1999 Jan & 15.4 & M5.5 \\
\hline GJ $1005 \ldots$ & $\mathrm{AB}$ & $0.1919 \pm 0.0172$ & 0.863 & 1992 Jan & 1999 Jan & 12.9 & M4.0 \\
\hline GJ $15 \ldots \ldots \ldots \ldots$ & $\mathrm{AB}^{\mathrm{a}}$ & $0.2802 \pm 0.0011$ & 2.912 & $1991 \mathrm{Aug}$ & 1999 Jan & 10.3 & M1.5 \\
\hline GJ $2005 \ldots \ldots \ldots \ldots$ & $\mathrm{ABCD}$ & $0.1328 \pm 0.0091$ & 0.614 & $\ldots$ & 1999 Jan & 15.4 & M5.5 \\
\hline GJ $54.1 \ldots \ldots \ldots \ldots$ & $\ldots$ & $0.2690 \pm 0.0076$ & 1.345 & $\ldots$ & 1999 Jan & 13.7 & M4.5 \\
\hline GJ 65 ..................... & $\mathrm{AB}$ & $0.3807 \pm 0.0043$ & 3.368 & 1992 Jan & 1999 Jan & 15.4 & M5.5 \\
\hline GJ $83.1 \ldots \ldots \ldots \ldots$ & $\ldots$ & $0.2238 \pm 0.0029$ & 2.907 & 1991 Aug & 1999 Jan & 14.0 & M4.5 \\
\hline GJ 109................ & $\ldots$ & $0.1324 \pm 0.0025$ & 0.923 & 1992 Jan & 1999 Jan & 11.2 & M3.0 \\
\hline GJ $185 \ldots \ldots \ldots \ldots \ldots$ & $\mathrm{AB}$ & $0.1203 \pm 0.0017$ & 0.308 & $1992 \mathrm{Feb}$ & 1999 Jan & 8.9 & K7.0 \\
\hline GJ 205................. & $\ldots$ & $0.1757 \pm 0.0012$ & 2.235 & 1992 Jan & $1998 \mathrm{Dec}$ & 9.1 & M1.5 \\
\hline GJ $213^{b} \ldots \ldots \ldots \ldots$ & $\ldots$ & $0.1728 \pm 0.0039$ & 2.571 & 1991 Oct & $1998 \mathrm{Dec}$ & 12.7 & M4.0 \\
\hline LHS $1805 \ldots \ldots \ldots . .$. & $\ldots$ & $0.1322 \pm 0.0029$ & 0.831 & $\ldots$ & $1998 \mathrm{Dec}$ & 12.3 & M3.5 \\
\hline G099-049 .......... & $\ldots$ & $0.1863 \pm 0.0062$ & 0.241 & . & $1998 \mathrm{Dec}$ & 12.7 & M3.5 \\
\hline GJ 229................ & $\mathrm{AB}$ & $0.1732 \pm 0.0011$ & 0.737 & 1992 Mar & $1998 \mathrm{Dec}$ & 9.3 & M1.0 \\
\hline GJ $234 \ldots \ldots \ldots \ldots$ & $\mathrm{AB}$ & $0.2429 \pm 0.0026$ & 0.997 & $1992 \mathrm{Feb}$ & $1998 \mathrm{Dec}$ & 13.0 & M4.5 \\
\hline GJ $251 \ldots \ldots \ldots \ldots$ & $\ldots$ & $0.1813 \pm 0.0019$ & 0.851 & 1992 Jan & 1998 Dec & 11.2 & M3.0 \\
\hline GJ $1093 \ldots \ldots \ldots \ldots$ & $\ldots$ & $0.1289 \pm 0.0035$ & 1.225 & $\ldots$ & 1999 Jan & 15.4 & M5.0 \\
\hline GJ 268 ................. & $\mathrm{AB}$ & $0.1572 \pm 0.0033$ & 1.052 & 1992 Jan & 1999 Jan & 12.5 & M4.5 \\
\hline GJ $273 \ldots \ldots \ldots \ldots$ & $\ldots$ & $0.2633 \pm 0.0014$ & 3.761 & 1992 Jan & 1999 Jan & 12.0 & M3.5 \\
\hline 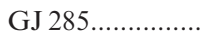 & $\ldots$ & $0.1686 \pm 0.0027$ & 0.604 & 1992 Mar & 1999 Jan & 12.3 & M4.0 \\
\hline GJ 299................ & $\ldots$ & $0.1480 \pm 0.0026$ & 5.211 & 1992 Mar & 1999 Jan & 13.7 & M4.0 \\
\hline GJ $300 \ldots \ldots \ldots \ldots \ldots$ & $\ldots$ & $0.1700 \pm 0.0102$ & 0.707 & 1992 Jan & & 14.2 & M3.5 \\
\hline GJ $1111 \ldots \ldots \ldots \ldots$ & $\ldots$ & $0.2758 \pm 0.0030$ & 1.29 & 1992 Jan & 1999 Jan & 17.0 & M6.5 \\
\hline GJ $1116 \ldots \ldots \ldots \ldots$ & $\mathrm{AB}$ & $0.1913 \pm 0.0025$ & 0.874 & 1992 Jan & 1999 Jan & 15.5 & M5.5 \\
\hline GJ 338.................. & $\mathrm{AB}^{\mathrm{a}}$ & $0.1616 \pm 0.0052$ & 1.662 & 1992 Jan & 1999 Jan & 8.7 & M0.0 \\
\hline GJ 380................ & $\ldots$ & $0.2052 \pm 0.0008$ & 1.454 & & 1999 Jan & 8.2 & K7.0 \\
\hline GJ 388 ................. & $\ldots$ & $0.2039 \pm 0.0028$ & 0.506 & $1992 \mathrm{Feb}$ & 1999 Jan & 11.0 & M3.0 \\
\hline GJ $393 \ldots \ldots \ldots \ldots$ & $\ldots$ & $0.1383 \pm 0.0021$ & 0.949 & 1992 Mar & 1999 Jan & 10.3 & M2.0 \\
\hline LHS $292 \ldots \ldots \ldots \ldots . . . .$. & $\ldots$ & $0.2210 \pm 0.0036$ & 1.644 & $1992 \mathrm{Feb}$ & 1999 Jan & 17.3 & M6.5 \\
\hline GJ $402 \ldots \ldots \ldots \ldots \ldots$ & $\ldots$ & $0.1775 \pm 0.0230$ & 1.15 & 1992 Mar & 1999 Jan & 12.9 & M4.0 \\
\hline GJ 406................. & $\ldots$ & $0.4183 \pm 0.0025$ & 4.696 & $1992 \mathrm{Mar}$ & 1999 Jan & 16.6 & M6.0 \\
\hline GJ 408................. & $\ldots$ & $0.1510 \pm 0.0016$ & 0.465 & 1992 Mar & 1999 Jan & 10.9 & M2.5 \\
\hline GJ 411................ & $\ldots$ & $0.3925 \pm 0.0009$ & 4.807 & 1992 Jan & 1999 Jan & 10.5 & M2.0 \\
\hline GJ $412 \ldots \ldots \ldots \ldots \ldots$ & $\mathrm{AB}^{\mathrm{a}}$ & $0.2069 \pm 0.0012$ & 4.528 & $1992 \mathrm{Mar}$ & 1999 Jan & 10.3 & M1.0 \\
\hline 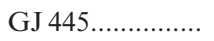 & $\ldots$ & $0.1855 \pm 0.0014$ & 0.863 & $1992 \mathrm{Feb}$ & 1999 Jan & 12.1 & M3.5 \\
\hline GJ 447................. & $\ldots$ & $0.2996 \pm 0.0022$ & 1.348 & 1992 Mar & 1999 Jan & 13.4 & M4.0 \\
\hline GJ $1156 \ldots \ldots \ldots \ldots . . . .$. & $\ldots$ & $0.1529 \pm 0.0030$ & 1.301 & & 1999 Jan & 14.7 & M5.0 \\
\hline GJ $473^{b} \ldots \ldots \ldots \ldots \ldots$ & $\mathrm{AB}$ & $0.2322 \pm 0.0043$ & 1.811 & 1992 Mar & $2000 \mathrm{Jan}$ & 14.3 & M5.5 \\
\hline GJ $514 \ldots \ldots \ldots \ldots \ldots$ & $\ldots$ & $0.1311 \pm 0.0013$ & 1.552 & 1992 Jan & 2000 Jan & 9.6 & M1.0 \\
\hline GJ $526 \ldots \ldots \ldots \ldots \ldots$ & $\ldots$ & $0.1841 \pm 0.0013$ & 2.325 & 1992 Mar & $2000 \mathrm{Jan}$ & 9.8 & M1.5 \\
\hline GJ $555^{b} \ldots \ldots \ldots \ldots$ & $\ldots$ & $0.1635 \pm 0.0028$ & 0.69 & 1992 Mar & 1998 Jul & 12.4 & M3.5 \\
\hline LHS $3003^{b} \ldots \ldots .$. & $\ldots$ & $0.1610 \pm 0.0060$ & 0.965 & $\ldots$ & 1998 Jul & 18.1 & M7.0 \\
\hline GJ $581^{\mathrm{b}} \ldots \ldots \ldots \ldots$ & $\ldots$ & $0.1595 \pm 0.0023$ & 1.224 & $1992 \mathrm{Mar}$ & $1998 \mathrm{Jul}$ & 11.6 & M2.5 \\
\hline GJ $623^{b} \ldots \ldots \ldots \ldots$ & $\mathrm{AB}$ & $0.1243 \pm 0.0012$ & 1.231 & 1991 Aug & $1998 \mathrm{Jul}$ & 10.7 & M2.5 \\
\hline 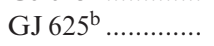 & $\ldots$ & $0.1519 \pm 0.0011$ & 0.42 & $1992 \mathrm{Mar}$ & 1998 May & 11.3 & M1.5 \\
\hline 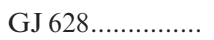 & & $0.2345 \pm 0.0018$ & 1.175 & 1992 Mar & $2000 \mathrm{Jul}$ & 12.0 & M3.0 \\
\hline GJ $644^{\mathrm{b}} \ldots \ldots \ldots \ldots \ldots$ & $\mathrm{ABCD}+643^{\mathrm{a}}$ & $0.1539 \pm 0.0026$ & 1.183 & 1992 Aug & 1998 May & 10.7 & M2.5 \\
\hline G203-047 ........... & $\mathrm{AB}$ & $0.1378 \pm 0.0090$ & 0.428 & $\ldots$ & 1998 May & 12.5 & M3.5 \\
\hline GJ $661^{\mathrm{b}} \ldots \ldots \ldots \ldots$ & $\mathrm{AB}$ & $0.1595 \pm 0.0031$ & 1.582 & 1991 Aug & 1998 May & 11.0 & M3.0 \\
\hline GJ $673 \ldots \ldots \ldots \ldots \ldots . . . .$. & $\ldots$ & $0.1295 \pm 0.0010$ & 1.315 & $\ldots$ & 1998 May & 8.1 & K7.0 \\
\hline GJ $686 \ldots \ldots \ldots \ldots . .$. & $\ldots$ & $0.1230 \pm 0.0016$ & 1.361 & $\ldots$ & 1998 May & 10.1 & M0.0 \\
\hline GJ 687................ & $\ldots$ & $0.2209 \pm 0.0009$ & 1.304 & $\ldots$ & 1998 May & 10.9 & M3.0 \\
\hline GJ 699 & $\ldots$ & $0.5493 \pm 0.0016$ & 10.31 & 1991 Aug & 1998 May & 13.2 & M4.0 \\
\hline GJ $701^{b} \ldots \ldots \ldots \ldots$ & $\ldots$ & $0.1283 \pm 0.0014$ & 0.644 & 1992 Aug & 1998 May & 9.9 & M0.0 \\
\hline GJ $1224 \ldots \ldots \ldots \ldots . .$. & $\ldots$ & $0.1327 \pm 0.0037$ & 0.664 & $\ldots$ & 1998 May & 14.3 & M4.5 \\
\hline LHS $3376 \ldots \ldots \ldots . .$. & $\ldots$ & $0.1373 \pm 0.0053$ & 0.623 & $\ldots$ & $1998 \mathrm{Jul}$ & 14.1 & M4.5 \\
\hline GJ $1230 \ldots \ldots \ldots \ldots . . . . . .$. & $\mathrm{ABC}$ & $0.1209 \pm 0.0072$ & 0.501 & 1991 Aug & 1998 May & 12.8 & M4.5 \\
\hline GJ $725 \ldots \ldots \ldots \ldots \ldots$ & $\mathrm{AB}^{\mathrm{a}}$ & $0.2802 \pm 0.0026$ & 2.273 & 1991 Oct & 1998 May & 11.1 & M3.0 \\
\hline GJ $729^{b}$. & $\ldots$ & $0.3365 \pm 0.0018$ & 0.72 & 1992 Aug & 1998 May & 13.6 & M3.5 \\
\hline GJ $752 \ldots \ldots \ldots \ldots \ldots$ & $\mathrm{AB}^{\mathrm{a}}$ & $0.1703 \pm 0.0014$ & 1.466 & 1992 Aug & 1998 May & 10.3 & M3.0 \\
\hline GJ $1245 \ldots \ldots \ldots \ldots$ & $\mathrm{AB}^{\mathrm{a} C}$ & $0.2120 \pm 0.0043$ & 0.731 & 1991 Aug & $1998 \mathrm{Jul}$ & 15.0 & M5.5 \\
\hline GJ $809 \ldots \ldots \ldots \ldots \ldots$ & $\ldots$ & $0.1420 \pm 0.0008$ & 0.772 & 1991 Oct & 2000 Nov & 9.3 & M0.0 \\
\hline 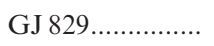 & $\mathrm{AB}$ & $0.1483 \pm 0.0019$ & 1.058 & 1991 Aug & 2000 Nov & 11.2 & M3.5 \\
\hline GJ $831 \ldots \ldots \ldots \ldots \ldots$ & $\mathrm{ABC}$ & $0.1256 \pm 0.0045$ & 1.194 & 1992 Aug & 2000 Nov & 12.6 & M4.5 \\
\hline
\end{tabular}


TABLE 1 - Continued

\begin{tabular}{|c|c|c|c|c|c|c|c|}
\hline $\begin{array}{c}\text { Primary Name } \\
\text { (1) }\end{array}$ & $\begin{array}{c}\text { Components } \\
\text { (2) }\end{array}$ & $\begin{array}{l}\text { Trig. Parallax } \\
\text { (3) }\end{array}$ & $\begin{array}{c}\text { Proper Motion } \\
\text { (4) }\end{array}$ & $\begin{array}{l}\text { First Epoch } \\
\text { (5) }\end{array}$ & $\begin{array}{l}\text { Second Epoch } \\
\text { (6) }\end{array}$ & $\begin{array}{l}M_{V} \\
\text { (7) }\end{array}$ & $\begin{array}{l}\text { Sp. Type } \\
\text { (8) }\end{array}$ \\
\hline LHS $3799 \ldots \ldots \ldots . . . .$. & $\ldots$ & $0.1341 \pm 0.0056$ & 0.778 & . & $2000 \mathrm{Nov}$ & 13.9 & M4.5 \\
\hline GJ $860 \ldots \ldots \ldots \ldots \ldots$ & $\mathrm{AB}$ & $0.2495 \pm 0.0030$ & 0.943 & 1991 Oct & 2000 Nov & 11.6 & M3.0 \\
\hline GJ 866.................. & $\mathrm{ABC}$ & $0.2943 \pm 0.0035$ & 3.254 & 1992 Aug & 2000 Nov & 14.4 & M5.0 \\
\hline GJ $873 \ldots \ldots \ldots \ldots$ & $\mathrm{AB}^{\mathrm{a}}$ & $0.1981 \pm 0.0021$ & 0.901 & 1991 Aug & $2000 \mathrm{Nov}$ & 11.6 & M3.5 \\
\hline GJ 876................ & $\mathrm{AB}$ & $0.2127 \pm 0.0021$ & 1.143 & 1992 Aug & 2000 Nov & 11.8 & M3.5 \\
\hline GJ $880 \ldots$ & $\ldots$ & $0.1453 \pm 0.0012$ & 1.071 & 1992 Aug & 2000 Nov & 9.5 & M1.5 \\
\hline GJ 884.................... & $\ldots$ & $0.1228 \pm 0.0009$ & 0.911 & $\ldots$ & 2000 Nov & 8.3 & K5.0 \\
\hline GJ 896.................. & $\mathrm{ABCD}$ & $0.1601 \pm 0.0028$ & 0.56 & 1992 Aug & 2000 Nov & 11.3 & M3.5 \\
\hline GJ 1286..................... & $\ldots$ & $0.1386 \pm 0.0035$ & 1.157 & 1992 Aug & $2000 \mathrm{Nov}$ & 15.4 & M5.5 \\
\hline GJ $905^{\mathrm{b}} \ldots \ldots \ldots \ldots \ldots$ & $\ldots$ & $0.3156 \pm 0.0016$ & 1.617 & 1991 Aug & 2000 Nov & 14.8 & M5.5 \\
\hline 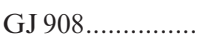 & $\ldots$ & $0.1675 \pm 0.0015$ & 1.37 & 1992 Aug & $2000 \mathrm{Nov}$ & 10.1 & M1.0 \\
\hline \multicolumn{8}{|c|}{ M Dwarfs That Would Now Meet Survey Criteria } \\
\hline 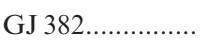 & $\ldots$ & $0.1273 \pm 0.0015$ & 0.287 & $\ldots$ & $\ldots$ & 9.3 & M1.5 \\
\hline G180-060 .......... & $\ldots$ & $0.1560 \pm 0.0040$ & $\ldots$ & $\ldots$ & $\ldots$ & 14.8 & M5.0 \\
\hline 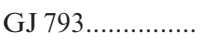 & $\ldots$ & $0.1251 \pm 0.0011$ & 0.526 & $\ldots$ & 1998 Jul & 10.4 & M2.5 \\
\hline LP $816-060 \ldots \ldots . .$. & $\ldots$ & $0.1822 \pm 0.0037$ & 0.308 & $\ldots$ & $2000 \mathrm{Nov}$ & 12.7 & $\mathrm{M}$ \\
\hline
\end{tabular}

Notes.-Column header explanations. Col. (1): Primary M star designation. Col. (2): Known companions. Col. (3): Trigonometric parallax (in arcseconds) from SHK96 or from Perryman et al. 1997. Col. (4): Proper motion of primary star (arcseconds per year). Col. (5): Date of first-epoch observation. Col. (6): Date of second-epoch observation. Col. (7): Absolute $V$-band magnitude of the primary star. Col. (8): Spectral type of the primary star.

a The companion was detected in the proper-motion search.

$\mathrm{b}$ The primary was not centered in the second-epoch image, so the search radius is not exactly 4.25.

Figure 3 shows the detection of the known low-mass companion VB 10 (GJ 752B). Other known wide companions were also detected as indicated in Table 1. These results demonstrate the reliability of the subtraction method. GJ 570A, known to have a T dwarf companion at a separation of 258.!3 (Burgasser et al. 2000), was not included in the sample because the primary is a $\mathrm{K} 4$ dwarf.
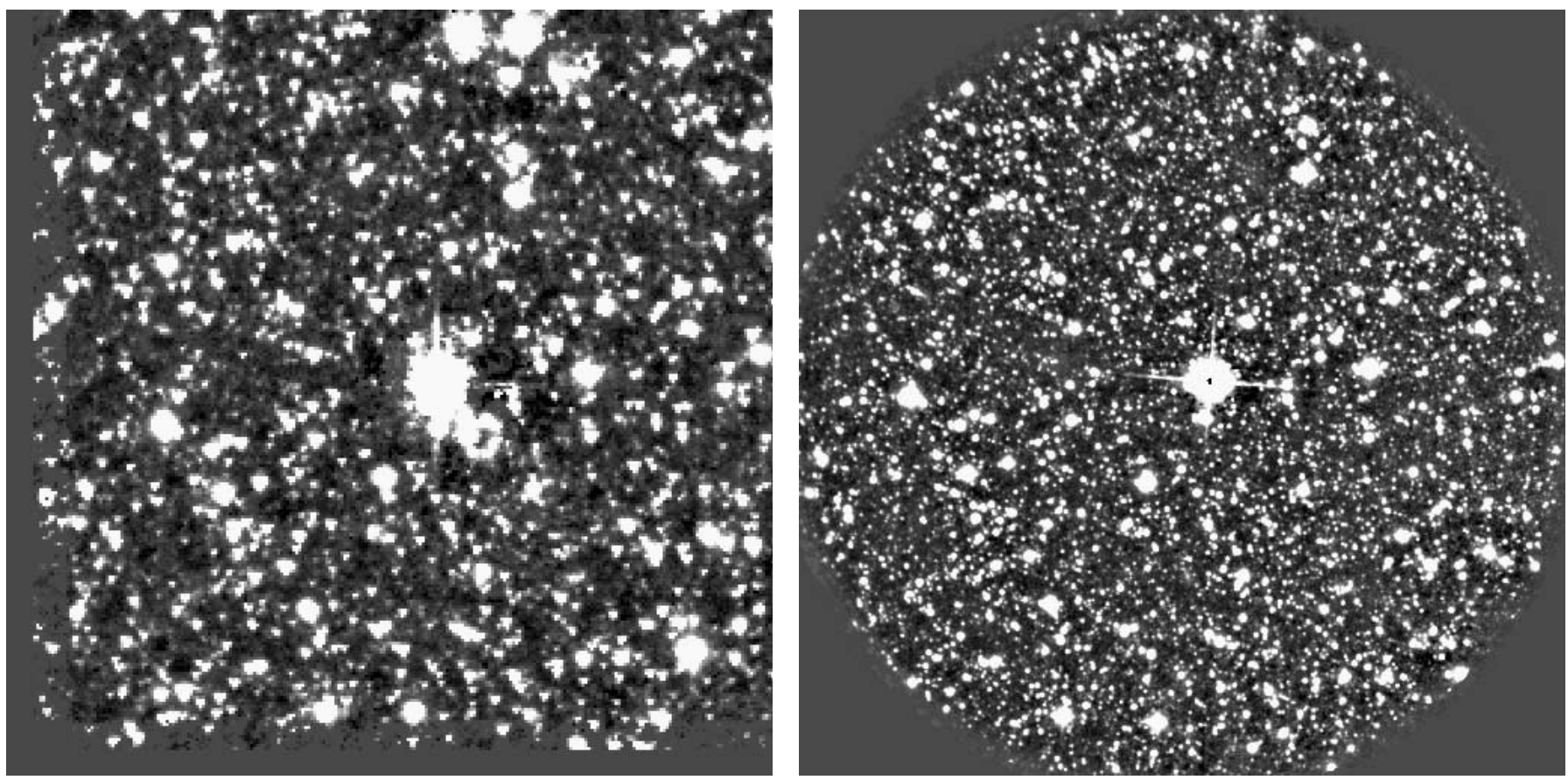

FIG. 2.-Sample $J$-band images of GJ 752 from the first (left) and second (right) epochs. North is up, and east is to the left. The field of view is 8.5 on a side, corresponding to a radial separation of 1500 AU from the primary star. The first-epoch image (SHK96) was obtained in 1992 and has a pixel scale of 2". 1 pixel $^{-1}$. The second-epoch image taken with the PISCES camera has a pixel scale of 0.5 pixel $^{-1}$. Limiting $J$-band magnitudes are $\sim 16.5$ and 17 , respectively. The ring of flux in the SHK96 image to the lower right of the primary star is an internal reflection in the optics of the infrared camera. 

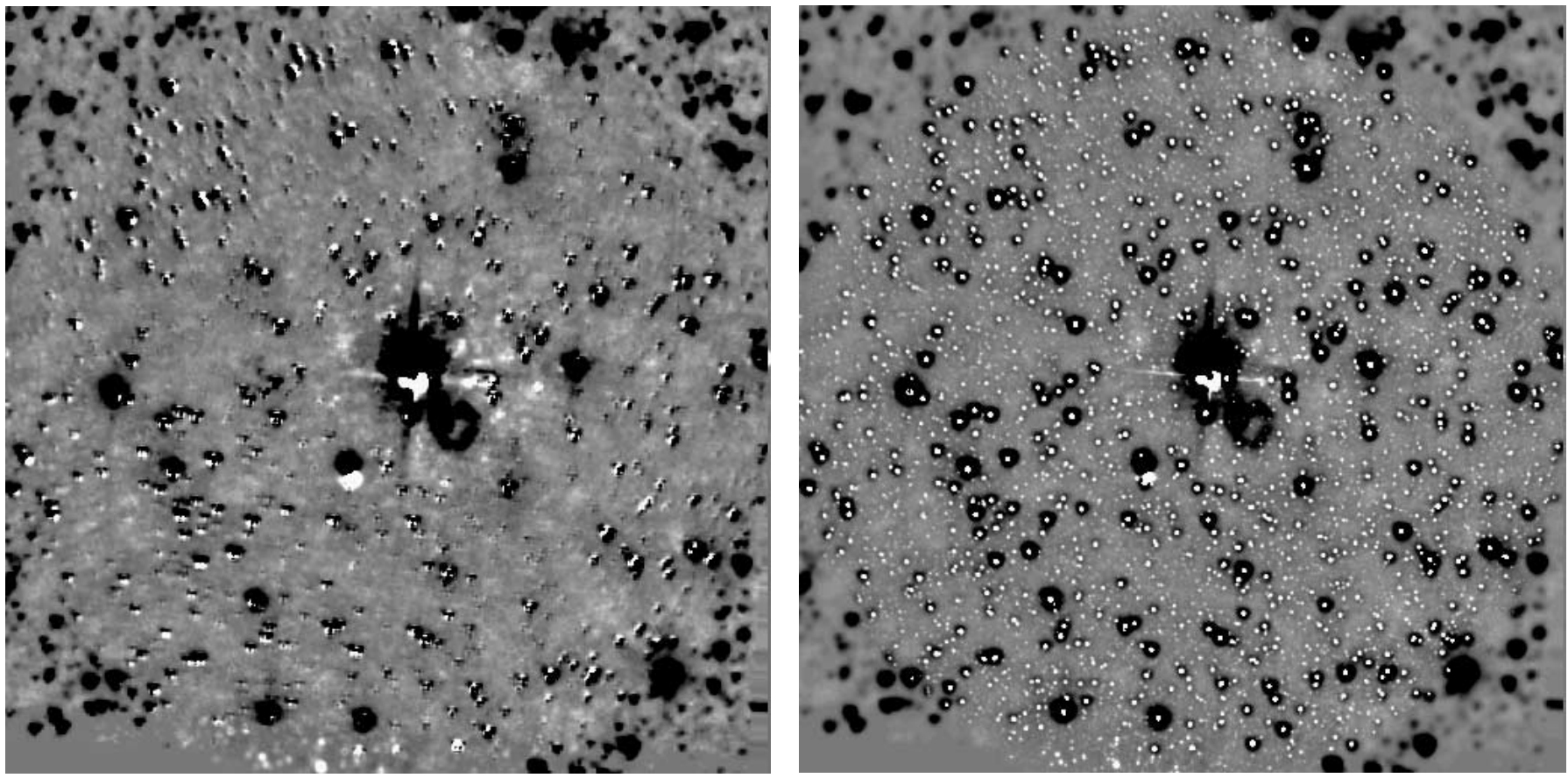

FIG. 3.-Difference images obtained by subtracting the first- and second-epoch images of GJ 752, as shown in Fig. 2. Results are shown with (left) and without (right) matching the point-spread functions of the two cameras before subtraction. The adjacent positive and negative images of the primary star show the motion over $\sim 6$ yr. The proper-motion companion VB 10, $m_{J} \sim 9.90$, is visible to the lower left of the primary star.

\section{RESULTS}

No new low-mass stellar or brown dwarf companions were detected in this 8 pc sample of $M$ dwarfs. The same conclusion was reached by SHK96 from a $J-K^{\prime}$ color search. However, as many as nine wide $(3600 \geq \Delta \geq 120$ AU) companions have recently been detected around nearby (9.6-39 pc) stars using the Two Micron All-Sky Survey (2MASS) database (Kirkpatrick et al. 2000, 2001; Burgasser et al. 2000; Wilson et al. 2001). Seven of these new objects are common proper-motion companions. In general, it is difficult to compare the 2MASS results with the present survey because the 2MASS primary stars have uncertain ages and generally higher luminosities. Based on initial 2MASS results, Gizis et al. (2001) estimate that $\sim 1 \%$ of primaries with masses $0.6-1.5 M_{\odot}$ $\left(M_{V}<9.5\right)$ have wide ( $\geq 1000$ AU) L dwarf companions and that the frequency of all wide brown dwarf companions is 5-13 times greater. Extending this analysis to our M dwarf sample of 63 objects with masses between 0.08 $0.6 M_{\odot}$, we would expect to detect between three $(5 \%$ of our sample) and nine (13\%) brown dwarf companions. The apparent difference between our results and Gizis et al. (2001) might be resolved if the frequency of brown dwarf companions depends strongly on primary mass and orbital separation. This possibility could be tested either by systematic data mining of the 2MASS survey or by extending the present PISCES survey to other spectral types.

J. L. H. thanks Chien Peng, Rose Finn, and Roelof de Jong for valuable discussions concerning near-infrared data reduction and Michael Meyer for comments on early drafts of this paper. The HAWAII detector and electronic controller for PISCES were purchased by the Air Force Office of Scientific Research through grant F49620-96-1-0285. The PISCES camera was also briefly supported by the National Science Foundation through grant NSF 96-23788. D. A. S. was supported by the Gemini Observatory, which is operated by the Association of Universities for Research in Astronomy, Inc., under a cooperative agreement with the National Science Foundation on behalf of the Gemini partnership: the National Science Foundation (US), the Particle Physics and Astronomy Research Council (UK), the National Research Council (Canada), CONICYT (Chile), the Australian Research Council (Australia), $\mathrm{CNPq}$ (Brazil), and CONICET (Argentina).
Barrado y Navascues, D., Stauffer, J. R., Bouvier, J., \& Martin, E. L. 2001, ApJS, 134, 103

Bouvier, J., Stauffer, J. R., Martin, E. L., Barrado y Navascues, D.,

Wallace, B., \& Bejar, V. J. S. 1998, A\&A, 336, 490

Burgasser, A., et al. 2000, ApJ, 531, L57

Burrows, A., et al. 1997, ApJ, 491, 856

Ducourant, C., Dauphole, B., Rapaport, M., Colin, J., \& Geffert, M. 1998, A\&A, 333, 882

Els, S. G., Sterzik, M. F., Marchis, F., Pantin, E., Endl, M., \& Kürster, M. 2001, A\&A, 370, L1

Gizis, J., Kirkpatrick, J. D., Burgasser, A., Reid, I. N., Monet, D. G., Liebert, J., \& Wilson, J. C. 2001, ApJ, 551, L163

\section{REFERENCES}

Gliese, W. 1969, Veröff. Astron. Rechen-Inst. Heidelberg, No. 22

Gliese, W., \& Jahreiss, H. 1979, A\&AS, 38, 423

Henry, T. 1991, Ph.D. thesis, Arizona Univ.

Kirkpatrick, J. D., Dahn, C. C., Monet, D. G., Reid, I. N., Gizis, J. E., Liebert, J., \& Burgasser, A. J. 2001, AJ, 121, 3235

Kirkpatrick, J. D., Reid, I. N., Gizis, J. E., Burgasser, A. J., Liebert, J.,

Monet, D. G., Dahn, C. C., \& Nelson, B. 2000, AJ, 120, 447

Lowrance, P. J., et al. 2000, ApJ, 541, 390 .1999, ApJ, 512, L69

Luhman, K. 2000, ApJ, 544, 1044

Luhman, K., Rieke, G. H., Lada, C. J., \& Lada, E. A. 1998, ApJ, 508, 347

Marcy, G. W., \& Butler, R. P. 1996, ApJ, 464, L147 
Mayor, M., \& Queloz, D. 1995, Nature, 378, 355

McCarthy, D. W., Jr., Ge, J., Hinz, J. L., Finn, R., \& de Jong, R. S. 2001, PASP, 113,353

Najita, J. R., Tiede, G. P., \& Carr, J. S. 2000, ApJ, 541, 977

Nakajima, T., Oppenheimer, B. R., Kulkarni, S. R., Golimowski, D. A., Matthews, K., \& Durrance, S. T. 1995, Nature, 378, 463

Oppenheimer, B. R., Golimowski, D. A., Kulkarni, S. R., Matthews, K., Nakajima, T., Creech-Eakman, M., \& Durrance, S. T. 2001, AJ, 121, 2189

Perryman, M. A. C., et al. 1997, A\&A, 323, L49
Reid, I. N., \& Gizis, J. E. 1997, AJ, 113, 2246

Reid, I. N., et al. 1999, ApJ, 521, 613

Reipurth, B., \& Clarke, C. 2001, AJ, 122, 432

Simons, D. A., Henry, T. J., \& Kirkpatrick, J. D. 1996, AJ, 112, 2238 (SHK96)

van Biesbroeck, G. 1961, AJ 66, 528

Van Dyk, S., Peng, C. Y., King, J. Y., Filippenko, A. V., Treffers, R. R., Li, W., \& Richmond, M. W. 2000, PASP, 112, 1532

Wilson, J. C., Kirkpatrick, J. D., Gizis, J. E., Skrutskie, M. F., Monet, D. G., \& Houck, J. R. 2001, AJ, 122, 1989 